\title{
Wandering spleen with chronic torsion in a patient with thalassaemia
}

Chi Long $\underline{\mathrm{Ho}}^{1}, \mathrm{MD}, \mathrm{FRCR}$

\begin{abstract}
Wandering spleen or splenoptosis is an uncommon entity and often an asymptomatic finding of acute abdomen in the emergency department. A high index of suspicion for splenic torsion is required, particularly in patients with known splenomegaly, as this condition could potentially lead to splenic infarction. Recognition of this condition can help avoid potential confusion with acute abdomen of other aetiologies. Herein, we present a unique case of wandering spleen with chronic torsion, which, to the best of our knowledge, has never been described in an elderly patient with haemoglobin $\mathrm{H}$ thalassaemia. We also review the literature for the aetiology and pathogenesis of wandering spleen, and discuss the relevant diagnostic modalities and treatment options.
\end{abstract}

Keywords: splenomegaly, splenoptosis, thalassaemia, torsion

\section{INTRODUCTION}

Wandering spleen, or splenoptosis, is an uncommon entity and often an incidental, asymptomatic finding of acute abdomen in the emergency department. The first case of wandering spleen was reported by Van Horne, a Dutch physician, during an autopsy in 1667. ${ }^{(1)}$ Wandering spleen is characterised by excessive mobility and displacement of the spleen of its normal location in the left hypochondrium, due to a lack of fixation and unduly long splenic pedicle. ${ }^{(2)}$ Recognition of this medical condition can help avoid any confusion with acute abdomen of other aetiologies. We herein present a unique case of an elderly woman with wandering spleen with a preponderance of chronic torsion, and review the literature for the aetiology, pathogenesis, relevant diagnostic modalities, and management options of splenoptosis.

\section{CASE REPORT}

A 66-year-old Chinese woman with a past history of haemoglobin $\mathrm{H}(\mathrm{HbH})$ thalassaemia was admitted to the gynaecology ward at the Singapore General Hospital, Singapore, under the clinical impression of an ovarian mass. She complained of intermittent abdominal pain and distension, which persisted for five years and increased in frequency over the last three months. On physical examination, she was afebrile, with a pulse rate of 88 beats per min, respiratory rate of 18 breaths per min, and blood pressure of $110 / 80 \mathrm{mmHg}$. She was oriented, cooperative and conscious. Her systemic examination was significant only for a hard, mobile mass (measuring $10 \mathrm{~cm} \times 10 \mathrm{~cm}$ ) in the left lower quadrant of the abdomen, extending into the pelvis.

Results of the laboratory tests were as follows: leucocyte

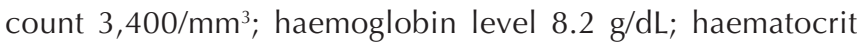
$24 \%$; platelet count $67,000 / \mathrm{mm}^{3}$; erythrocyte sedimentation rate $10 \mathrm{~mm} / \mathrm{hr}$; C-reactive protein level $0.5 \mathrm{mg} / \mathrm{L}$; negative direct Coombs' and indirect Coombs' test; glucose level $180 \mathrm{mg} / \mathrm{dL}$; sodium $141 \mathrm{mEq} / \mathrm{L}$; potassium $3.9 \mathrm{mEq} / \mathrm{L}$; calcium $8.9 \mathrm{mg} / \mathrm{dL}$; urea $34 \mathrm{mg} / \mathrm{dL}$; creatinine $0.7 \mathrm{mg} / \mathrm{dL}$; total bilirubin $1.32 \mathrm{mg} / \mathrm{dL}$; direct

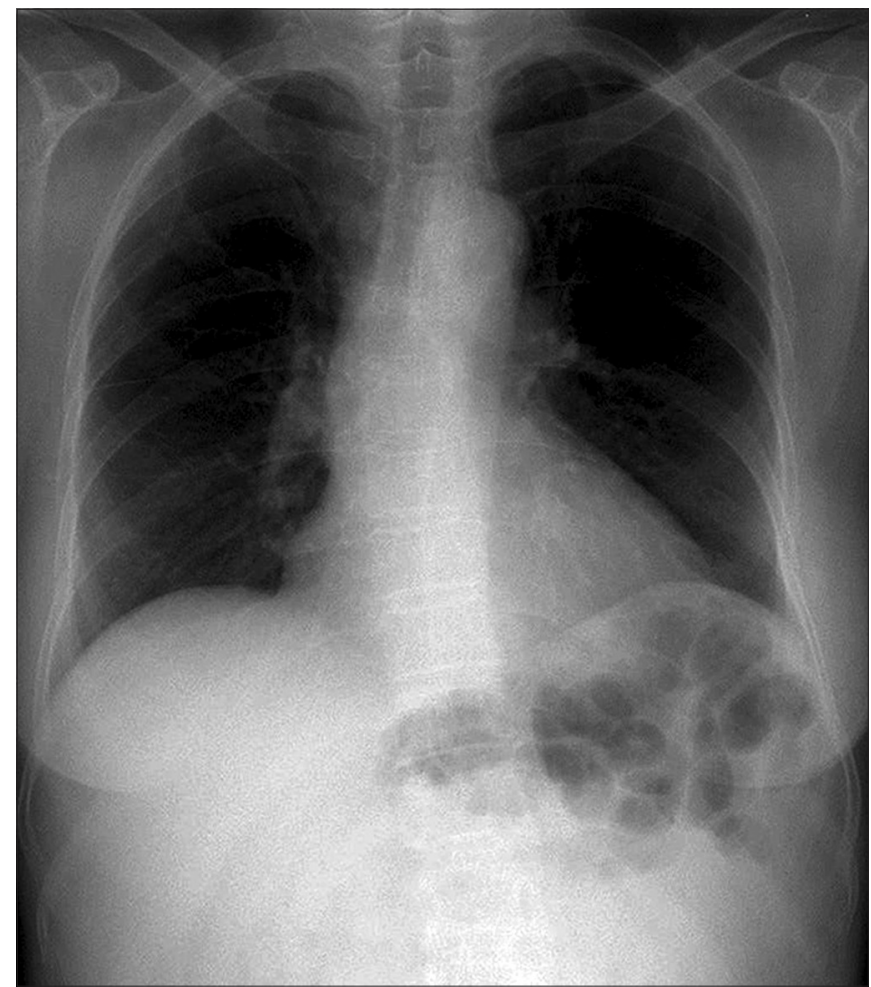

Fig. 1 Chest radiograph reveals bowel loops underneath the left hemidiaphragm and absence of a splenic silhouette in an otherwise clear pair of lungs.

bilirubin $0.4 \mathrm{mg} / \mathrm{dL}$; aspartate aminotransferase $17 \mathrm{U} / \mathrm{L}$; alanine aminotransferase $15 \mathrm{U} / \mathrm{L}$; and gamma glutamyl transpeptidase $20 \mathrm{U} / \mathrm{L}$. The peripheral blood film revealed hypochromia and microcytosis without iron deficiency. Haemoglobin analysis by liquid chromatography revealed the presence of $\mathrm{HbH}$ inclusion bodies.

On plain radiography, bowel loops were seen underneath the left hemidiaphragm, with the absence of a splenic silhouette (Fig. 1). Computed tomography (CT) of the abdomen and pelvis showed the absence of the spleen in its normal position (i.e. the 

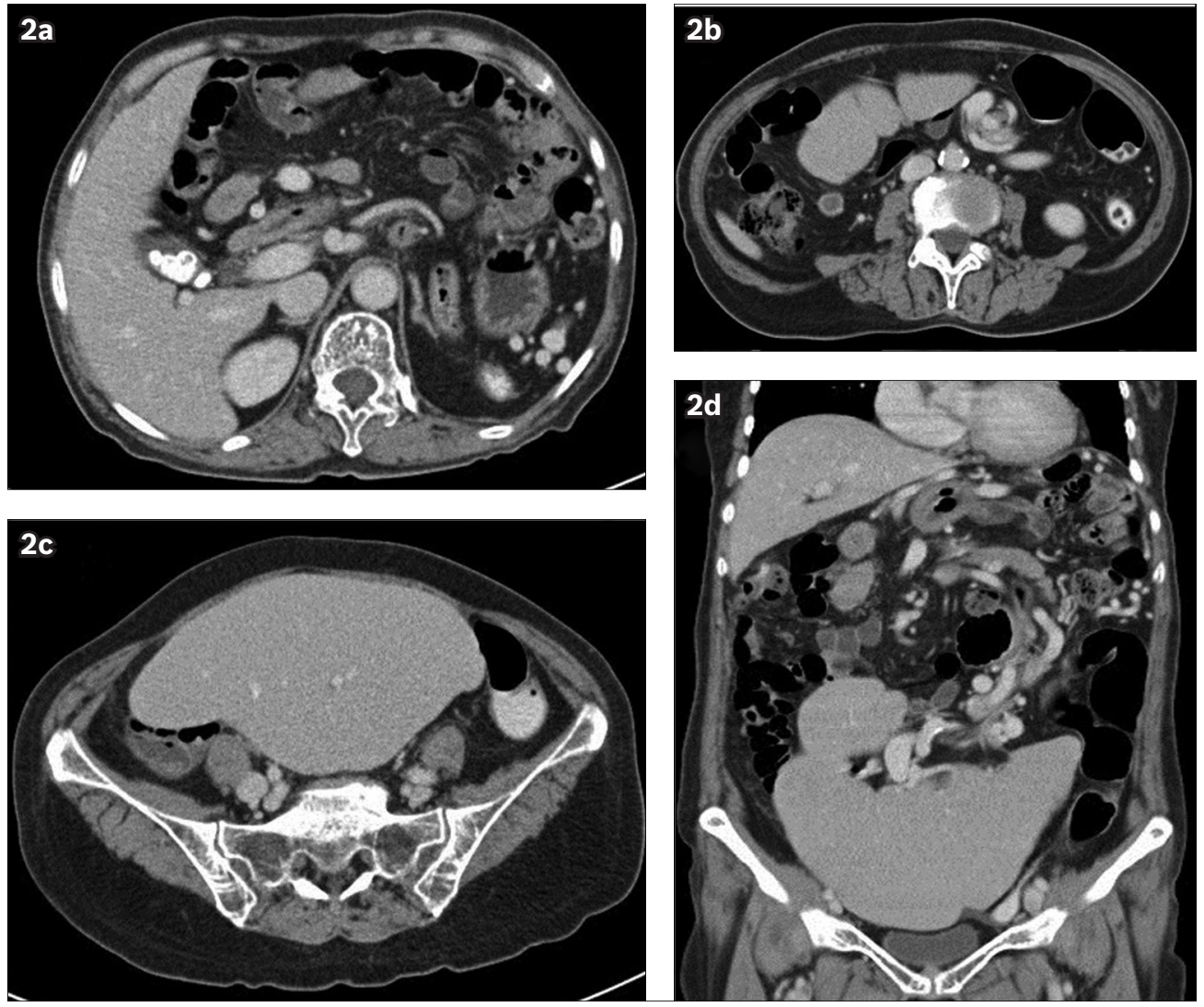

Fig. $2(\mathrm{a}-\mathrm{c})$ Contrast-enhanced axial CT images of the abdomen and pelvis demonstrate the 'whorl' sign of the long vascular pedicle extending to the abnormally positioned spleen. There is no fat stranding surrounding the twisted splenic pedicle, and contrast enhancement of the splenic parenchyma is preserved with no evidence of infarction. Gallstones are also noted. (d) Contrast-enhanced coronal reconstructed CT image demonstrates a lobulated, enlarged spleen, which lies in the pelvis and lower abdomen, with an elongated splenic pedicle in torsion.

left hypochondrium) (Figs. 2a \& b). There was a downward displacement of an enlarged, lobulated-appearing spleen in the lower abdomen and upper pelvic cavity (Figs. 2c \& d). The splenic pedicle was twisted in a 'whorl' configuration (Figs. $2 b$ \& d). Notwithstanding the splenic torsion, there was no splenic infarction, insofar as the splenic contrast enhancement was clearly homogeneous.

Red blood cell transfusion was required for our anaemic patient upon her admission. Surgery with an option for splenopexy was offered to her but she declined. Her symptoms subsequently subsided following a short period of observation in the ward before being discharged from the hospital. She remained asymptomatic in subsequent follow-ups in the clinics.

\section{DISCUSSION}

The spleen is surrounded by peritoneal attachments such as the gastrosplenic ligament, which forms part of the splenic anchoring ligaments from the splenic hilum to the greater curvature of the stomach, carrying the short gastric and left gastroepiploic vessels. $^{(2)}$ The lienorenal ligament, which passes from the spleen to the left kidney, carries the splenic vessels with it along the pancreatic tail. ${ }^{(2)}$ Splenoptosis is defined as a mobile spleen that is attached only by an elongated vascular pedicle, allowing it to migrate to any part of the abdomen or pelvis. ${ }^{(3}$

During fetal development, the dorsal mesogastrium may fail to fuse with the posterior peritoneum, leading to the laxity or absence of splenic-supporting ligaments. ${ }^{(4,5)}$ The aetiology of this splenic hypermobility may be congenital or acquired. Congenital splenoptosis is characterised by the absence or underdevelopment of one or both of the aforementioned anchoring ligaments. ${ }^{(4,5)}$ Most cases of congenital splenoptosis occur with a male to female ratio of $6: 1$ in children younger than one year of age. ${ }^{(6,7)}$ Meanwhile, acquired splenoptosis can occur in young adults or adolescents, caused by underlying conditions that result in laxity of the ligamentous support, such as hormonal effects of pregnancy, abdominal wall laxity and splenomegaly. ${ }^{(8,9)}$ Interestingly, splenoptosis occurred in our 66-year-old thalassaemic female patient. Splenomegaly due to various other causes, including haematological diseases (chronic myeloid leukaemia and lymphoma), infections (including parasitic infections such as kala-azar and malaria), and metabolic disorders (Gaucher's disease and Niemann-Pick disease) can also result in elongation of the splenic pedicle by traction and subsequently increase the chance of occurrence of splenic torsion. ${ }^{(8,10-12)}$

Torsion of the wandering spleen is a rare phenomenon, with an incidence of 1 in 2,000 and accounts for only 2 per 1,000 splenectomies. ${ }^{(3,4)}$ Acute splenic torsion usually occurs in a clockwise rotation and can lead to vascular congestion, infarction, and even gangrene of the spleen. ${ }^{(6,7)}$ It presents as an acute abdomen and can mimic peritonitis, acute appendicitis, twisted ovarian cyst, diverticulitis, cholecystitis or bowel obstruction. ${ }^{(13)}$ 
Imaging studies are helpful in establishing the diagnosis of wandering spleen and to differentiate it from other causes of acute abdomen. Abdominal radiography may reveal the absence of a splenic silhouette, with bowel loops seen closely abutting the left upper flank. Abdominal ultrasonography is useful in establishing the absence or presence of the spleen in the left upper quadrant and localising the abnormal position of the spleen. ${ }^{(14)}$ A corresponding duplex study can assess the splenic blood flow and the viability of splenic parenchyma. ${ }^{(14)}$ Splenoptosis should be distinguished from ectopic spleen, a condition where the splenic tissue develops at an abnormal site, and also from splenosis, asplenia and accessory spleen. ${ }^{(2,3)}$ Another consideration for the absence of the spleen from its normal position is polysplenia, in which numerous small splenic masses are scattered in the abdomen and this can be associated with abdominal situs and cardiovascular anomalies. ${ }^{(3)}$

Abdominal CT is considered the imaging investigation of choice for acute abdomen. Imaging features may vary with the stages of the splenic infarction, which can be acute or chronic. ${ }^{(15)}$ In the hyperacute phase of splenic infarction, CT may show areas of mottled, increased attenuation, representing areas of haemorrhagic infarction. ${ }^{(5)}$ In splenic torsion, which leads to acute infarction, CT would reveal a marked fat stranding in the region of the splenic hilum near the whirling of the splenic vessels due to venous congestion. ${ }^{(16)}$ The 'rim' sign of the high density splenic capsule compared with lower attenuation of the compromised splenic parenchyma is another $\mathrm{CT}$ finding of splenic infarction; the occurrence of the sign probably a result of collateral circulation to the splenic capsule. ${ }^{(4,5,17)}$ In our patient, the 'whorl' sign observed on CT was suggestive of torsion, but there was a lack of fat stranding surrounding the twisted splenic pedicle to suggest venous congestion and impending acute infarction. ${ }^{(17)}$ One of the possible explanations for this phenomenon is spontaneous detorsion of the splenic pedicle, thus allowing splenic venous return via the dilated and serpiginous gastric vessels along the greater and lesser curvatures of the stomach, before emptying into the commencement of the main portal vein. ${ }^{(17-19)}$ Splenic infarction in the chronic phase may even disappear completely, although more commonly, it demonstrates progressive volume loss caused by fibrotic contraction of the infarcted areas and compensatory hypertrophy of the surrounding normal splenic parenchyma. ${ }^{(9,18)}$ Such compensatory hypertrophy of the splenic parenchyma can explain the lobulated appearance of the enlarged spleen in our patient with chronic torsion. In addition to revealing splenoptosis, CT also shows the viability of splenic parenchyma, as well as the anatomic relationships and associated pathologies of the surrounding viscera.

The rare association between gastric volvulus, diaphragmatic hernia and wandering spleen have been reported previously, as these conditions share a common cause, which is the absence or laxity of intraperitoneal visceral ligaments. ${ }^{(3,4,18,19)}$ The other common causes reported are necrosis with involvement of the pancreatic tail and its surrounding fat due to the torsion of splenic vessels. ${ }^{(3,18,19)}$ Magnetic resonance imaging is a rarely used modality for acute abdomen, as the imaging technique may show splenic infarction as peripheral wedge-shaped defects that exhibit decreased signal intensity on both T1- and T2-weighted images with no enhancement following the administration of intravenous contrast material.

Splenopexy is the procedure of choice to prevent future torsion while preserving the functional splenic tissue when a viable wandering spleen is found at surgery. ${ }^{(10,13,20)}$ Splenectomy should only be performed when there is no evidence of viable splenic parenchyma after detorsion of the spleen. ${ }^{(10,13,20)}$

In conclusion, wandering spleen with torsion poses a great diagnostic challenge of acute abdomen in the emergency department due to the rarity of its occurrence. It requires a very high index of suspicion, particularly in patients with known splenomegaly, as this condition could potentially lead to splenic infarction.

\section{REFERENCES}

1. Lane TM, South LM. Management of a wandering spleen. J R Soc Med 1999; 92:84-5.

2. Qazi SA, Mirza SM, Muhammad AM, Al Arrawi MH, Al-Suhaibani YA. Wandering spleen. Saudi J Gastroenterol 2004; 10:1-7.

3. Liu HT, Lau KK. Wandering spleen: an unusual association with gastric volvulus. AJR Am J Roentgenol 2007; 188:W328-30.

4. Spector JM, Chappell J. Gastric volvulus associated with wandering spleen in a child. J Pediatr Surg 2000; 35:641-2.

5. Romero J, Barksdale EM Jr. Wandering spleen: a rare cause of abdominal pain. Pediatr Emerg Care 2003; 19:412-4.

6. Zimmermann ME, Cohen RC. Wandering spleen presenting as an asymptomatic mass. Aust N Z J Surg 2000; 70:904-6.

7. Horwitz JR, Black CT. Traumatic rupture of a wandering spleen in a child: case report and literature review. J Trauma 1996; 41:348-50.

8. Paterson A, Frush DP, Donnelly LF, et al. A pattern-oriented approach to splenic imaging in infants and children. Radiographics 1999; 19:1465-85.

9. Taori K, Ghonge N, Prakash A. Wandering spleen with torsion of vascular pedicle: early diagnosis with multiplanar reformation technique of multislice spiral CT. Abdom Imaging 2004; 29:479-81.

10. Magowska A. Wandering spleen: a medical enigma, its natural history and rationalization. World J Surg 2013; 37:545-50.

11. Weatherall DJ. Genetic variation and susceptibility to infection: the red cell and malaria. Br J Haematol 2008; 141:276-86.

12. McFee RB, Musacchio T, Gorgescu D, et al. Wandering spleen with torsion in a geriatric patient. Report of an unusual case with a brief review of the clinical picture and management. Dig Dis Sci 1995; 40:2656-9.

13. Dawson JH, Roberts NG. Management of the wandering spleen. Aust N Z J Surg 1994; 64:441-4.

14. Nemcek AA Jr, Miller FH, Fitzgerald SW. Acute torsion of a wandering spleen: diagnosis by CT and duplex Doppler and color-flow sonography. AJR Am J Roentgenol 1991; 157:307-9.

15. Ityel H, Granot $Y$, Vaknine H, Judich A, Shimonov M. Splenic torsion of a wandering spleen. Isr Med Assoc J 2011; 13:119-20.

16. Ben Ely A, Zissin R, Coppel L, et al. The wandering spleen: CT findings and possible pitfalls in diagnosis. Clin Radiol 2006; 61:954-58.

17. Collie DA, Ferguson JM, Allan PL, Redhead DN. Case report: computed tomography features of complete splenic infarction, cavitation and spontaneous decompression complicating pancreatitis. Br J Radiol 1995; 68:662-4.

18. Rodkey ML, Macknin ML. Pediatric wandering spleen: case report and review of literature. Clin Pediatr 1992; 31:289-94.

19. Pelizzo G, Lembo MA, Franchella A, et al. Gastric volvulus associated with congenital diaphragmatic hernia, wandering spleen, and intrathoracic left kidney: CT findings. Abdom Imaging 2001; 26:306-8.

20. Corcione F, Caiazzo P, Cuccurullo D, et al. Laparoscopic splenectomy for the treatment of wandering spleen. Surg Endosc 2004; 18:554-6. 